\title{
Qualidade das informações sobre fecundidade no Censo Demográfico de 2010
}

\author{
Suzana M. Cavenaghi* \\ José Eustáquio Diniz Alves **
}

O objetivo desta nota de pesquisa é examinar a qualidade da informação sobre fecundidade no Censo Demográfico brasileiro de 2010, para inferir sobre a precisão das estimativas da taxa de fecundidade total (TFT) e das taxas específicas por idade provenientes dessa fonte de dados. As bases de dados utilizadas são os microdados do Censo Demográfico de 2010 e o Sistema de Informações sobre Nascimentos (Sinasc) de 2009 e 2010. Serão aplicados indicadores demográficos básicos e o método indireto da razão $\mathrm{P} / \mathrm{F}$ de Brass para a estimação da TFT, utilizando as duas fontes de dados. Os resultados mostram que os dados censitários são bastante consistentes, mas apresentam alguns problemas de subenumeração por idade da fecundidade corrente, como amplamente conhecido na demografia. Esta subenumeração deve ser levada em consideração na estimação do nível de fecundidade no país. As mudanças na estrutura da fecundidade afetam a TFT por ser taxa de período, mas o método P/F de Brass ainda mostra bons resultados. Ao final, são feitas recomendações para possíveis melhorias em novas coletas de dados, principalmente nos censos e pesquisas por amostra de domicílios, que são fontes essenciais para a estimação da fecundidade e acompanhamento de suas tendências, necessárias para a atualização das projeções desse indicador no médio e longo prazos.

Palavras-chave: Qualidade da informação. Fecundidade. Métodos indiretos. Brasil.

\footnotetext{
* Escola Nacional de Ciências Estatísticas (Ence), Instituto Brasileiro de Geografia e Estatística (IBGE), Rio de Janeiro-RJ, Brasil (suzana_cavenaghi@uol.com.br).

** Escola Nacional de Ciências Estatísticas (Ence), Instituto Brasileiro de Geografia e Estatística (IBGE), Rio de Janeiro-RJ, Brasil (jed_alves@yahoo.com.br).
} 


\section{Introdução}

Na década de 1960, a necessidade de se obterem estimativas de indicadores demográficos em países com dados deficientes estimulou o desenvolvimento de métodos indiretos para estimação das taxas de fecundidade e de mortalidade, entre outras. Nos últimos 50 anos, a demografia da América Latina tem se valido desses métodos, que sofreram pequenos ajustes, e continua a contar com eles, apesar de as taxas de fecundidade e mortalidade já terem alcançado níveis baixos. A implementação de sistemas de registro civil que forneçam dados de qualidade e oportunos tem sido uma história de muitos desafios na região. Assim, ainda que pareça um contrassenso para demógrafos de países desenvolvidos, em nossa região os dados censitários são fulcrais, principalmente para as estimativas das taxas de fecundidade.

No caso do Brasil existe uma longa tradição na coleta de informações sobre fecundidade e mortalidade nos censos demográficos, mesmo antes dos anos 1960. A partir do Censo Demográfico de 1940, foi incluída a pergunta sobre total de filhos nascidos vivos e sobreviventes para as mulheres com 15 anos ou mais de idade (e dez ou mais a partir de 1991). Em 1970 foi incluída a informação sobre filhos nascidos no ano anterior (12 meses) à pesquisa. Desde então, a utilização desses dados, principalmente com o emprego das técnicas indiretas, baseadas nos dados de parturição (P) e fecundidade corrente (F), tem sido recorrente no país para as estimativas da taxa de fecundidade total (TFT), ou seja, a taxa de período (UNITED NATIONS, 1983). Outros métodos indiretos, que também utilizam dados censitários, mas não com o uso das perguntas sobre fecundidade acumulada e corrente, como o método dos filhos próprios (CHO, 1973), foram empregados por alguns autores (WONG, 1983; FERNANDEZ; CARVALHO, 1986), mas de maneira muito esporádica, assim como métodos para criação de histórias de nascimentos a partir dos dados censitários para estimativas dos efeitos de quantum e tempo da TFT (MIRANDA-RIBEIRO; RIOS-NETO; CARVALHO, 2013). Portanto, tanto no Brasil como na maioria dos países latino-americanos, os censos demográficos são fontes essenciais para estimativas de taxas de fecundidade, principalmente informações oficiais e aquelas usadas nas projeções populacionais. Assim, mesmo após o país ter atingido o nível de fecundidade abaixo da reposição, os métodos baseados, primordialmente, nas informações sobre parturição e fecundidade corrente ainda são muito utilizados, apesar de não estarem isentos de críticas.

Desde os trabalhos de William Brass sempre foi bastante conhecido que os dados sobre fecundidade corrente nos censos demográficos (ou em pesquisas domiciliares), em geral, são subestimados para todos os grupos etários, mas as evidências do passado mostravam que esta subestimação não era diferencial por idade das mulheres, fato que levou ao estabelecimento de cálculos da TFT com os ajustes da fecundidade corrente (F) a partir da fecundidade acumulada (P) das mulheres jovens (UNITED NATIONS, 1983), conhecido como ajustes a partir das análises das razões $\mathrm{P} / \mathrm{F}$. 
Logo após a divulgação dos dados censitários de 2010, houve muita discussão sobre a qualidade da informação sobre fecundidade, em função da constatação de que os fatores de ajustes (razões $\mathrm{P} / \mathrm{F}$ ) tinham aumentado em comparação, principalmente, ao Censo Demográfico de 2000. A primeira pergunta que se fazia era se a nova forma de captar a informação, a partir da implementação do questionário em formato digital, com mudança na ordem das perguntas do bloco de fecundidade (bloco em forma vertical), priorizando a pergunta e não a entrevistada, tinha afetado o resultado da coleta de dados. Ainda, se mudanças na estrutura da fecundidade podiam estar afetando de alguma forma as razões $P / F$, devido a uma possível postergação da fecundidade para os grupos de 15-19 e 20-24 anos. Mais recentemente, alguns autores levantaram a hipótese de inadequação do uso do método P/F para ajustar a fecundidade em 2010 (CASTANHEDA; KOLHER, 2016), não somente no Brasil, mas também em outros países latino-americanos, com a hipótese de que a TFT é mais baixa do que os valores resultantes da aplicação do método P/F de Brass com o ajuste para mulheres de 20-24 anos de idade.

Paralelamente, outros autores (MOULTRIE et al., 2013) afirmam que o método P/F de Brass está ultrapassado e deve ser substituído por outros métodos paramétricos, como o relacional de Gompertz, também inicialmente proposto por Brass, que não assume o pressuposto de fecundidade constante em período recente (somente que as mudanças são graduais e afetam todas as idades igualmente). Partindo da convicção (e evidências) de que os métodos que utilizam dados de parturição e fecundidade corrente ainda são os mais recomendados para o Brasil (com a escolha do devido fator de correção, não necessariamente P/F de 20-24 anos), Berquó e Cavenaghi (2014) proporcionam uma comparação entre o método $\mathrm{P} / \mathrm{F}$ de Brass e as variações do método relacional de Gompertz. A conclusão das autoras é que, para cálculo da TFT para grupos populacionais com estruturas da fecundidade distintas (por exemplo, urbano e rural, por educação, etc.), o método paramétrico ainda não oferece melhores estimativas do que o $\mathrm{P} / \mathrm{F}$ de Brass, que não tem curva de fecundidade implícita. Ao contrário, o método de Gompertz, na forma apresentada atualmente, mostra distorções importantes nas taxas específicas de fecundidade por idade, principalmente para seguimentos populacionais com baixa e rejuvenescida fecundidade.

Em outra vertente, a utilização dos dados censitários para calcular a TFT se justifica pela cobertura diferencial das informações do Registro Civil no país, que, apesar dos enormes esforços realizados pelos produtores de dados, ainda apresentava problemas de cobertura em algumas regiões até os anos 1990. A despeito da grande melhoria na última década na cobertura dos registros administrativos sobre nascimentos, o Registro Civil e o Sistema de Informações de Nascimentos (Sinasc) ainda possuem problemas em alguns municípios (cobertura diferencial por regiões, estados e municípios). Além disso, o fato de esses registros terem poucas informações socioeconômicas, as quais permitiriam avançar nos estudos sobre os diferenciais de fecundidade, é outro motivo que torna os dados do censo demográfico (e outras pesquisas domiciliares) tão atraentes. 
De todo modo, é importante buscar o estabelecimento de evidências sobre a qualidade/consistência dos dados de fecundidade no Censo Demográfico 2010 a partir de indicadores demográficos básicos, bem como pela comparação desses com os dados dos registros administrativos de nascimentos, e obter indícios sobre a cobertura dessa informação no censo. Logo, o objetivo desta nota é examinar a qualidade da informação sobre a fecundidade corrente no Censo Demográfico 2010 e a possível influência que este pode apresentar no nível e padrão da fecundidade declarada. Para finalizar, são apresentadas algumas recomendações sobre o módulo de fecundidade para os censos demográficos e pesquisas domiciliares.

\section{Dados e métodos}

A principal fonte de dados utilizada é o Censo Demográfico 2010, com as informações provenientes do módulo de fecundidade e mortalidade, referentes ao total de filhos nascidos vivos (FNV) e filho nascido no último ano (FNUA), que é identificado a partir da pergunta sobre data (mês e ano) de nascimento do último filho nascido vivo. ${ }^{1}$ Os dados podem ser classificados por idade das mulheres e fornecem, portanto, informações sobre fecundidade acumulada (parturição) e fecundidade corrente (F) por idade.

É importante destacar que nos censos demográficos brasileiros, desde 1980, é incluída a informação sobre o sexo dos FNV (também para filhos nascidos mortos e sobreviventes) e, a partir de 1991, o sexo dos FNUA. Esse levantamento, além de permitir melhor qualidade na coleta das informações, pois funciona como um mecanismos para recordar alguma criança que porventura tenha ficado de fora das declarações, também possibilita calcular indicadores de qualidade dos dados coletados, como a razão de sexo dos nascidos no último ano, que será apresentada neste trabalho.

Sobre o censo demográfico brasileiro, é importante destacar alguns aspectos metodológicos que podem afetar a qualidade das informações. Duas características são distintas da maioria dos censos de população dos países latino-americanos. A primeira delas, por questões operacionais em um país com as dimensões territoriais e populacionais do Brasil, é ter um questionário pequeno aplicado ao universo da população, sendo que a maior parte dos temas, incluindo fecundidade, é inserida em outro questionário aplicado a uma amostra que tem variado a fração amostral ao longo dos censos. ${ }^{2}$ Este questionário da amostra é bastante extenso e variou de tamanho no decorrer dos anos (em 2010 apresentou 108 perguntas), com o módulo de fecundidade alocado no final (CAVENAGHI; ALVES, 2011). A

\footnotetext{
${ }^{1}$ Em 1940 e 1950, o censo demográfico era aplicado a toda a população e não incluiu a informação sobre fecundidade do último ano. Em 1960 o censo passou a ter uma parte amostral e realizado com entrevistas diretas. Em 1970 a pergunta sobre número de filhos nascidos nos 12 meses anteriores à data de referência do censo (noite de 30 de agosto para 1 de setembro) foi incluída e permaneceu, com diferentes formas de perguntar, até o censo atual. As informações eram coletadas para mulheres de 15 anos ou mais e a, partir de 1991, para aquelas com dez anos ou mais.

${ }^{2} 0$ tamanho amostral, nos censos de 1960 e 1980, foi de 25\% dos domicílios; em 1991 e 2000 foram duas frações amostrais, uma de $10 \%$ e outra de $20 \%$ (em municípios com menos de 170 mil habitantes) e, em 2010 , cinco frações amostrais, variando de $5 \%$ a $50 \%$ segundo o tamanho do município (IBGE, 2013a).
} 
segunda característica é que o levantamento dos dados censitários realiza-se durante três a quatro meses e, portanto, o período de referência para algumas perguntas, assim como ser um censo de fato ou de direito (definição de quem são os moradores de cada domicílio), passa a ser atributo essencial para garantir a qualidade dos dados.

A segunda fonte utilizada para comparação com os dados do censo demográfico é o Sistema de Informações de Nascidos Vivos (Sinasc), para 2009 e 2010, mantido pelo Ministério da Saúde, com dados coletados nos estabelecimentos hospitalares (cerca de $98 \%$ dos nascimentos registrados ocorrem em hospitais). ${ }^{3}$ Ainda, são empregadas as projeções populacionais por sexo e idade, para mulheres em idade reprodutiva, considerando a revisão das projeções de 2013 (IBGE, 2013b).

$O$ método principal utilizado neste trabalho é o método indireto da razão $P / F$, proposto por Brass (1973), com as variações introduzidas ao método posteriormente. Um dos pressupostos do método é que a fecundidade tenha permanecido constante em anos recentes, mas a fecundidade em declínio não afeta sobremaneira as estimativas como mostrado para o caso brasileiro (CARVALHO, 1982; BERCOVICH, 1986), principalmente quando as idades mais jovens são consideradas para o ajuste das taxas. Dado o extenso conhecimento deste método na literatura, não se expõem aqui os seus detalhes (UNITED NATIONS, 1983). Vale também mencionar que, recentemente, novos desenvolvimentos na área de estimação da taxa total de fecundidade e seleção do fator de ajuste têm sido propostos (SCHEMERTMAN, 2012; SCHEMERTMAN; CAVENAGHI; ASSUNÇÃO; POTTER, 2013), mas para os propósitos desta nota o método $\mathrm{P} / \mathrm{F}$ de Brass se mostrou suficiente.

\section{Resultados}

\section{A qualidade da informação sobre fecundidade corrente no censo de 2010}

As informações declaradas sobre fecundidade corrente em pesquisas domiciliares, com entrevistas realizadas na maioria das vezes com proxies (um informante para todos os membros do domicílio), quando bem coletadas, apresentam boa declaração, não registram problemas graves de memória, mas sim no que diz respeito a períodos de referência, quando a informação é coletada por período e não por data de nascimento do filho mais novo (UNITED NATIONS, 1983). Esta curva da fecundidade corrente declarada nos censos brasileiros tem apresentado uma distribuição suave, para as idades simples, sendo que em 2010 esta distribuição para as idades de 10 a 49 anos (Gráfico 1) mostra que os dados têm boa qualidade, com pequenas altercações nas idades da mãe de 25 e 30 anos e com o pico na de 21 anos.

\footnotetext{
$\overline{3}$ O Sinasc foi escolhido para a análise por apresentar os dados de uso público em formato de microdados, diferente do Registro Civil, que somente disponibiliza dados agregados para uso público. No entanto, sabe-se que em algumas localidades o Registro Civil tem melhor cobertura das informações (quando somados registros tardios) do que o Sinasc, mas em outras localidades o Sinasc registra melhor cobertura.
} 
GRÁFICO 1

Taxas específicas de fecundidade corrente declarada $\left(f_{x}\right)$, por idade simples das mães Brasil - 2010

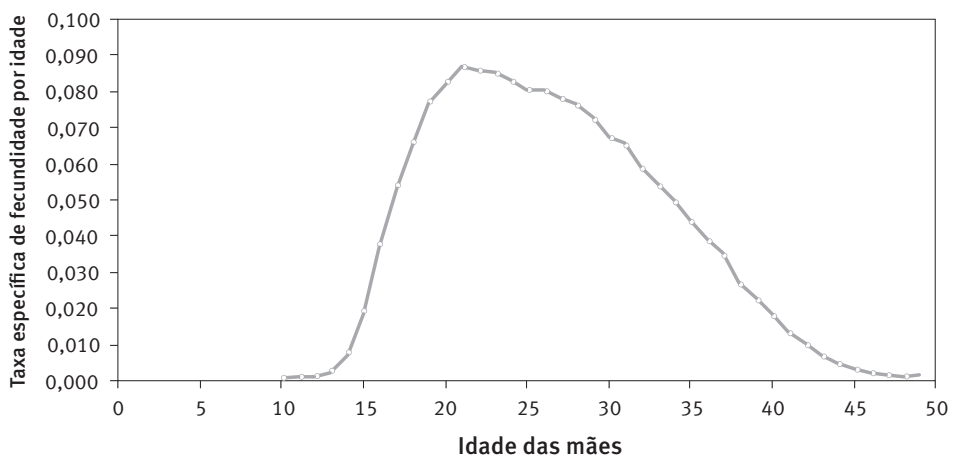

Fonte: IBGE. Microdados do Censo Demográfico de 2010.

A razão de sexo ao nascimento apresenta, em geral, valores esperados ao redor de 105 nascimentos de meninos para cada 100 nascimentos de meninas. Em 2010, as estimativas da razão de sexo dos filhos nascidos nos 12 meses anteriores ao censo mostram exatamente o valor de 105,2, quando consideradas as mulheres de 15 a 49 anos, ou mesmo se incluídas aquelas de 10 a 14 anos. A distribuição por idade simples desta razão de sexo ao nascimento, no entanto, como mostrado no Gráfico 2, tem uma distribuição bastante irregular, com valores que variam de menos de 80 meninos para cada 100 meninas até 120 . As maiores variações ocorrem para as mulheres de 10 a 19 anos. É importante ressaltar que este resultado pode ser devido ao valor baixo de número total de filhos nascidos no ano anterior para estas mulheres, que é de 21.076 crianças (valor populacional expandido), entre os mais de 2,6 milhões de nascimentos ocorridos no período. Entretanto, mesmo considerando o grupo de mulheres de 15 a 49 anos, a curva oscila ao redor do valor de 105 e tem tendência a diminuição conforme aumenta a idade das mulheres. Ou seja, para mulheres com 45 anos ou mais, parece que nasce mais meninas do que meninos. Além do baixo número de nascimentos nestas idades (13.018 nascimentos no ano anterior), os dados podem indicar a ocorrência de alguma seletividade (natural ou não) de sexo ao nascimento, favorecendo nascimentos femininos para este grupo etário, ou, ainda, pode indicar problemas de confundimento entre o termo nascido vivo e nascido morto, com diferencial por sexo, para as mulheres neste grupo etário.

A irregularidade da distribuição da razão de sexo ao nascimento por idade das mães é suavizada ao se observar esta mesma distribuição por grupos de idades quinquenais (Gráfico 2). Este resultado pode ser característico de problemas na declaração de idade das mulheres e não de erro na declaração da fecundidade. Quanto ao nível da razão de sexo dos nascimentos, este se mantém ao redor do esperado: 105 para todos os grupos quinquenais, exceto para o último, de 45 a 49 anos de idade, em que nascem mais meninas do que meninos (93 meninos para cada 100 meninas), ou seja, a variação deste grupo não é contrarrestada com o uso da média do grupo de idade quinquenal. Dessa forma, pode-se 
concluir que a distribuição da fecundidade atual para grupos quinquenais oferece uma boa distribuição desta variável para os dados do Censo Demográfico de 2010.

\section{GRÁFICO 2}

Razão de sexo de nascidos nos 12 meses anteriores à realização do censo, por idade das mães (idade simples e grupos quinquenais)

Brasil - 2010

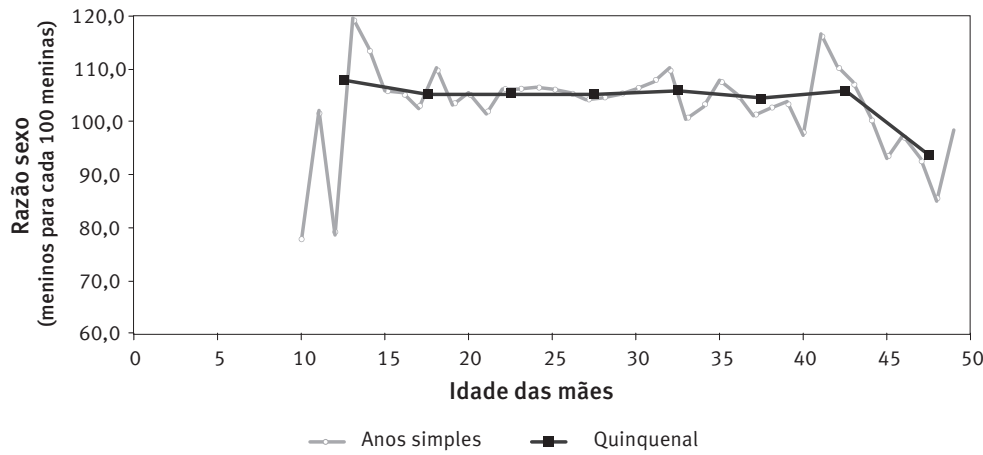

Fonte: IBGE. Microdados do Censo Demográfico de 2010.

Nota: Observar que o eixo do $x$ começa em 60.

No Censo Demográfico de 2010, o questionário disponibilizado não previu, para a pergunta sobre filhos nascidos vivos, sobreviventes ou nascido no último ano (mês e ano do nascimento do último filho nascido vivo ou idade do último filho nascido vivo), a alternativa não sabe (ou ignorado). ${ }^{4}$ Assim, na base de microdados a informação sobre ignorados também não está disponível. No entanto, no censo de 2000, estes casos eram reduzidos, com menos de $1 \%$ em todos os grupos etários, ${ }^{5}$ visto que sofreram crítica e, posteriormente, imputação de zero filhos para as situações em que se podia identificar que a não resposta correspondia de fato a mulheres sem filhos. Dessa forma, quanto à distribuição por idade, as indicações são de que o Censo Demográfico de 2010 apresenta boa qualidade da informação sobre fecundidade corrente $\left(f_{x}\right)$. Na próxima seção buscam-se indicações sobre 0 grau de cobertura da $f_{x}$, o que determina o nível da fecundidade declarada.

\footnotetext{
4 Deve-se lembrar, no entanto, que as entrevistas no Censo Demográfico de 2010 foram realizadas com o PDA e o questionário disponibilizado é meramente ilustrativo. Para certificar-se sobre como a questão dos dados ignorados no tema de fecundidade foi tratada, é necessário ter acesso ao aplicativo com o questionário digital.

${ }^{5}$ Uma análise sobre a porcentagem de não resposta aos dados de fecundidade nos Censos Demográficos de 1970, 1980 e 1991 foi realizada por Cavenaghi (1999), em que a autora mostra que a porcentagem de mulheres com fecundidade ignorada variou ao longo dos anos. Principalmente, identificou que foram corrigidos os dados para o grupo de mulheres de 15 a 19 anos, em 1970, para o grupo de 15 a 24 anos, em 1980, e não houve nenhuma correção, em 1991. Para o Brasil em 1970, a porcentagem máxima de não resposta foi de $6 \%$ para mulheres com 20 a 24 anos, reduzindo até $2 \%$ para aquelas de 35 a 49 anos. Em 1980, esta distribuição teve seu máximo no grupo de 25 a 29 anos, com $4 \%$ de não reposta, mas permaneceu ao redor de 3\% nos grupos etários seguintes. Em 1991, como não teve correção, o máximo de não resposta ocorre para o grupo de 15 a 19 anos (7,5\%) e decresceu até 2,5\% e 3,0\% nos grupos seguintes (CAVENAGHI, 1999, p. 130-134).
} 


\section{O nível da fecundidade corrente}

As informações do Censo Demográfico de 2010 têm como data de referência 31 de julho. ${ }^{6}$ A Tabela 1 mostra a distribuição do número de filhos nascidos no ano anterior ao censo por idade quinquenal das mães, ou seja, nascimentos ocorridos entre agosto de 2009 e julho de 2010. Para obter informações mais comparáveis com esta, disponibilizam-se os dados do registro administrativo como média de todos os nascimentos ocorridos durante 2009 e 2010, tendo como meio do período o início de 2010. Pode-se observar que os resultados do censo apontam um menor número de nascimentos do que no registro administrativo, no total e para todos os grupos etários até 34 anos. A diferença é de 7,7\%, totalizando 220 mil nascimentos a menos captados pelo censo. No entanto, percebe-se que a maior diferença está no grupo de 15 a 19 anos, seguido pelo de 20 a 24 anos, enquanto nos grupos de 35 anos ou mais o comportamento é inverso, com o registro administrativo apresentando menor número de nascimentos do que o censo.

O número de mulheres por idade quinquenal, no censo demográfico e nas projeções populacionais, que serve como denominador das taxas de fecundidade, no entanto, apresenta pouquíssimas diferenças, em que se esperaria mais, visto que a cobertura do censo demográfico raramente chega perto de $100 \%$. Comparações realizadas na conciliação censitária para a revisão das projeções de população de 2013 (IBGE, 2013) mostram que a população com melhor cobertura no censo são as mulheres em idade reprodutiva, o que justificaria, portanto, esta pequena diferença entre dados projetados e população enumerada.

TABELA 1

Número de nascimentos nos registros administrativos e censo demográfico e número de mulheres nas projeções e no censo, segundo grupos de idade das mulheres

Brasil - 2010

\begin{tabular}{|c|c|c|c|c|c|c|c|c|}
\hline \multirow{3}{*}{$\begin{array}{l}\text { Grupos } \\
\text { etários }\end{array}$} & \multicolumn{4}{|c|}{ Nascimentos } & \multicolumn{4}{|c|}{ Mulheres } \\
\hline & \multicolumn{2}{|c|}{$\begin{array}{c}\text { Registro } \\
\text { administrativo } \\
\text { (média 2009-2010) } \\
\end{array}$} & \multicolumn{2}{|c|}{$\begin{array}{l}\text { Censo de } 2010 \\
\text { (FNUA) }\end{array}$} & \multicolumn{2}{|c|}{$\begin{array}{l}\text { Estimativa anual em } \\
2010\end{array}$} & \multicolumn{2}{|c|}{ Censo 2010} \\
\hline & $\mathrm{N}$ & $\%$ & $\mathrm{~N}$ & $\%$ & $\mathrm{~N}$ & $\%$ & $\mathrm{~N}$ & $\%$ \\
\hline 15 a 19 anos & 536.270 & 18,9 & 417.496 & 15,9 & 8.432 .002 & 15,7 & 8.407 .461 & 15,7 \\
\hline 20 a 24 anos & 804.971 & 28,3 & 727.638 & 27,7 & 8.614 .963 & 16,1 & 8.587 .420 & 16,0 \\
\hline 25 a 29 anos & 718.709 & 25,3 & 670.351 & 25,6 & 8.643 .418 & 16,1 & 8.620 .668 & 16,1 \\
\hline 30 a 34 anos & 488.498 & 17,2 & 478.461 & 18,2 & 8.026 .855 & 15,0 & 8.006 .350 & 15,0 \\
\hline 35 a 39 anos & 231.581 & 8,1 & 242.291 & 9,2 & 7.121 .916 & 13,3 & 7.103 .781 & 13,3 \\
\hline 40 a 44 anos & 59.752 & 2,1 & 74.245 & 2,8 & 6.688 .797 & 12,5 & 6.672 .250 & 12,5 \\
\hline 45 a 49 anos & 4.104 & 0,1 & 13.017 & 0,5 & 6.141 .338 & 11,4 & 6.126 .957 & 11,4 \\
\hline Total & 2.843 .884 & 100,0 & 262.3499 & 100,0 & 53.669 .289 & 100,0 & 53.524 .887 & 100,0 \\
\hline
\end{tabular}

Fonte: Sinasc 2009 e 2010; Censo Demográfico de 2010 e IBGE (2013b), projeções populacionais.

\footnotetext{
${ }_{6}$ Importante registrar que, desde o censo de 2000, as perguntas do bloco de fecundidade e mortalidade incluem a data de referência do censo na própria pergunta, visto que o censo demora até quatro meses para ser realizado. (Ex.: “Quantos filhos e filhas nascidos vivos teve até 31 de julho de 2010?” ). Este procedimento tem potencialidade para melhorar a resposta obtida, no entanto, não se conhecem publicações sobre testes realizados sobre este assunto.
} 
Diferenças entre as distribuições de nascimentos por idade das mães são esperadas ao se compararem os dados do registro e do censo, visto que a idade das mães, no primeiro, é a do momento do nascimento e, nos censos, é aquela no momento da pesquisa. ${ }^{7}$ Esta diferença pode ser observada no Gráfico 3, que traz a distribuição relativa das taxas de fecundidade, principalmente para o primeiro grupo quinquenal de idade. 0 painel à direita no Gráfico 3, que apresenta os dados da estrutura etária da fecundidade corrigida para grupos convencionais de idade, mostra que as duas fontes de informação têm praticamente a mesma estrutura, com uma pequena diferença no grupo de 20 a 24 anos. Este fato corrobora a observação feita por Brass (1961) de que a estrutura da fecundidade corrente (FNUA) é de boa qualidade.

As taxas de fecundidade específicas por idade estimadas com informações das duas fontes de dados, apresentadas no painel inferior do Gráfico 3, mostram, como observado na distribuição do número de nascimentos (Tabela 1), que o nível obtido com os dados de FNUA subestima a fecundidade. Se ainda for considerado que os registros administrativos não alcançam 100\% dos nascimentos, pode-se afirmar que os dados do censo de 2010 apresentam subestimação importante. A taxa de fecundidade total, a partir das duas fontes de dados, se não forem corrigidos os níveis de sub-registro, seria de 1,72 e 1,60 filho por mulher, respectivamente para o Sinasc e o censo. Dessa forma, os dados indicam que é necessário estimar um fator de ajuste que eleve o nível da fecundidade corrente quando se utilizam os dados do censo.

O método proposto por Brass (UNITED NATIONS, 1983) para corrigir a fecundidade corrente $(\mathrm{F})$ proveniente da pergunta sobre FNUA nas pesquisas domiciliares, como amplamente conhecido, é compará-la com a fecundidade acumulada $(\mathrm{P})$ e ajustar o nível utilizando as razões $\mathrm{P} / \mathrm{F}$ das idades mais jovens, em geral 20 a 29 anos. 0 Gráfico 4 mostra as séries de $\mathrm{P} / \mathrm{F}$ para os últimos quatro censos brasileiros. Todos os valores são maiores do que um, o que significa que a fecundidade corrente está subestimada ou está declinando. Para idades mais avançadas no período reprodutivo, é de se esperar que esteja realmente ocorrendo uma queda nas taxas de fecundidade por idade. Para as mulheres mais jovens, observa-se que o grupo de 20 a 24 anos apresentou valores próximos de $12 \%$ até 2000 , aumentando para quase $20 \%$ no censo seguinte. De fato, a curva das razões P/F para 2010 apresenta-se acima da curva dos demais censos (exceto 35 anos e mais para 1991).

\footnotetext{
7 Por exemplo, uma mulher de 15 anos de idade pode ter tido o filho no início do período ou já no final. Ou seja, o filho poderia ter apenas um dia de vida ou estar por completar um ano. Se a probabilidade de que as mulheres tenham filhos de 1 a 12 meses é uniformemente distribuída, pode-se dizer que estas mulheres tiveram filhos quando tinham em média 14,5 anos ou 15,5 anos. Assim, a idade das mulheres ao terem seus filhos, e não a idade no momento da pesquisa, corresponde a grupos de 14,5-18,5 anos (chamados de grupos quinquenais não convencionais) e não ao grupo de $15-19$ anos como no registro de nascimentos (UNITED NATIONS, 1983).
} 


\section{GRÁFICO 3}

Estrutura da fecundidade atual declarada/registrada e corrigida e taxas específicas de fecundidade $\left(f_{\mathrm{x}}\right)$, por grupos de idade quinquenais convencionais, segundo fonte de dados

\section{Brasil - 2010}
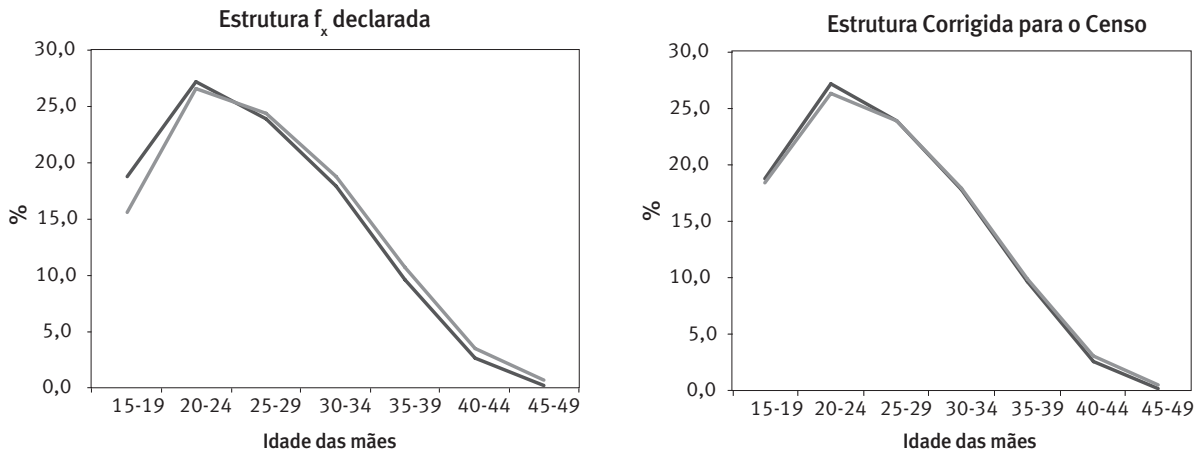

- Sinasc 2009-10 - Censo 2010

- Sinasc 2009-10 - Censo 2010
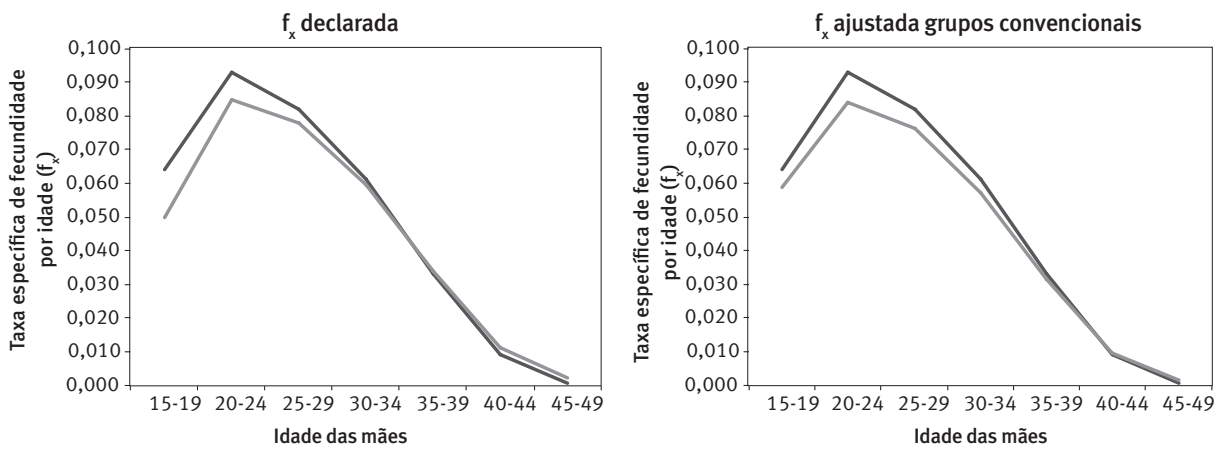

- Sinasc 2009-10 - Censo 2010

- Sinasc 2009-10 _ Censo 2010

Fonte: Sinasc 2009 e 2010; Censo Demográfico de 2010 e IBGE (2013b), projeções populacionais.

GRÁFICO 4

Séries das razões $\mathrm{P} / \mathrm{F}$ (parturição e fecundidade corrente equivalentes), por grupos de idade das mães Brasil - 1980-2010

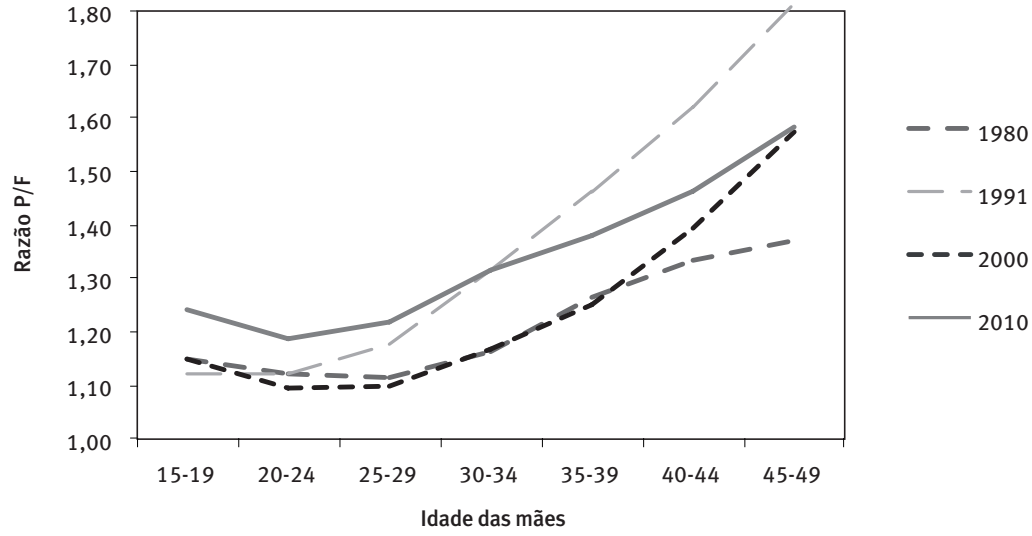

Fonte: IBGE, Censos Demográficos de 1980, 1991, 2000 e 2010.

Nota: Razões calculadas pelo método P/F de Brass e ajustes de Trussel (UNITED NATIONS, 1983). 
O censo de 1991, que registra uma curva muito distinta a partir dos 25 anos de idade, teve as perguntas separadas para filhos nascidos vivos que estavam vivos, fazendo referência àqueles que moravam no domicílio e aqueles que moravam em outro domicílio. No entanto, em 2010, que também mostra uma curva bastante diferente das demais, a pergunta foi similar à de 2000. De fato, ao se compararem as perguntas censo a censo, em todos houve alguma mudança na forma ou na ordem das questões. Em 2010, ocorreu uma alteração importante na sequência das perguntas, que, em vez de coletar para cada entrevistada todas as suas informações, fez-se a mesma pergunta para todas as entrevistadas (questionário no computador de mão com desenho vertical).

Outro fator que pode alterar o formato da curva, além da forma de se elaborar a pergunta, da ordem das perguntas, da subestimação diferencial da cobertura de cada censo e do ritmo de queda da fecundidade, é o fato de haver alguma postergação ou antecipação de nascimentos, ou seja, uma mudança na estrutura da fecundidade. Em 2010, observa-se que o processo de rejuvenescimento da estrutura da fecundidade que vinha acontecendo nos anos anteriores cessou e, inclusive, aparece uma pequena postergação para mulheres acima de 35 anos. Assim, na comparação do P/F para o grupo de 20 a 24 anos, por exemplo, pode estar refletido um $\mathrm{F}$ menor devido a uma possível postergação de alguns nascimentos e não, de fato, em função de uma mudança no número final de filhos. Isso causa uma alteração momentânea na TFT que é uma medida de período, mas não necessariamente na taxa de fecundidade que representaria a experiência de uma coorte. Se este é o caso, a fecundidade corrente coletada no censo de 2010 não necessitaria de um aumento de $19 \%$ para que a parturição do grupo de 20 a 24 anos fosse coerente à fecundidade corrente deste grupo, visto que realmente ocorreria uma queda momentânea devido à postergação de alguns nascimentos.

Se o ajuste da fecundidade for aplicado utilizando o valor de P/F do grupo de 20 a 24 anos de idade, o nível da TFT declarada no censo, de 1,60 filho por mulher, aumenta para 1,90, quando o nível da TFT registrada no Sinasc é de 1,72, empregando dados de 2009 e 2010 e a população feminina estimada para o meio do período. No entanto, como sabe-se que o Sinasc também não tem cobertura de $100 \%$ em todo território nacional, a aplicação do método $\mathrm{P} / \mathrm{F}$ para ajustar esta fecundidade também seria recomendada. Para isso, além da população estimada de mulheres, é necessário usar os dados de parturição coletados no censo demográfico. Com este procedimento, a TFT estimada com dados de fecundidade corrente do Sinasc seria de 1,84 filho por mulher (Gráfico 5). Portanto, conclui-se que a estimativa da TFT a partir dos dados do Censo Demográfico de 2010 precisa do ajuste de cerca de $19 \%$ para um valor mais próximo do nível "verdadeiro". Assim, a comparação destes dados indica que a fecundidade pode estar próxima de 1,9 e 1,84 filho por mulher, mas o verdadeiro valor não tem como ser conhecido, visto que o registro é deficiente e a fecundidade de período, calculada pelo censo, pode estar afetada por mudanças conjunturais na estrutura da fecundidade e erros ou supostos que não se cumprem.

Ainda, deve-se ter em mente que a TFT calculada com o fator de ajuste referente às mulheres de 20 a 24 anos fornece uma fecundidade média para os últimos 2,5 anos (algo 
entre 2008 e julho de 2010) e não exatamente para 2010. Dessa forma, ainda que seja válido buscar outros métodos de estimação como alguns autores vêm propondo, todas estas medidas terão seus próprios erros embutidos e serão somente estimativas, sem se poder afirmar qual o valor mais correto da TFT. Soma-se a isso o fato de que a TFT é uma medida de período que provavelmente não se efetivará no futuro, visto que há fortes indícios de continuada queda da fecundidade no Brasil.

GRÁFICO 5

Taxa de fecundidade total (1), segundo fontes de dados utilizadas Brasil - 2010

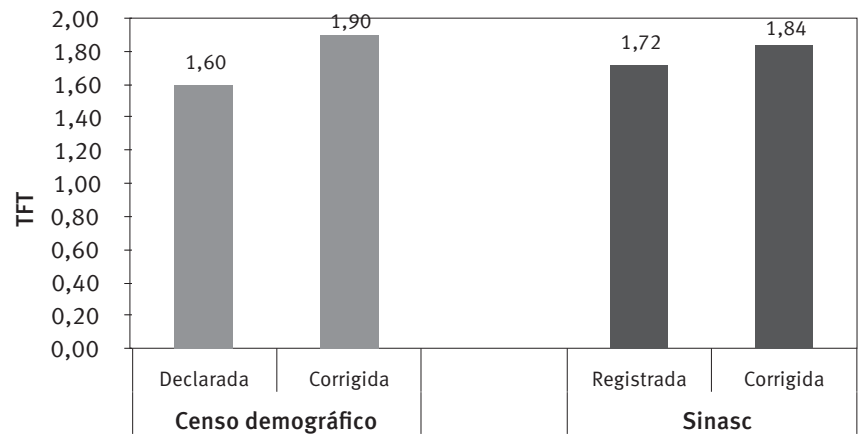

Fonte: IBGE (2013b), projeções populacionais e Censo Demográfico de 2010. Sinasc 2009 e 2010. (1) Número médio de filhos por mulher.

\section{Algumas considerações para a melhoria da coleta da informação sobre fecundidade nos censos demográficos}

Algumas mudanças e ampliações na forma de coleta sobre fecundidade nos próximos censos seria essencial para manter a qualidade da informação e melhoria na coleta. Deve-se ter em mente que esta informação é essencial para entender sobre as tendências da fecundidade, novos padrões e possíveis indicações do comportamento futuro da fecundidade que afeta sobremaneira o ritmo de crescimento populacional e, portanto, essencial para boas projeções populacionais.

Com relação à mudança de formato do questionário, com a introdução do PDA, seria importante conhecer melhor o procedimento adotado em 2010 e fazer testes de campo para saber mais sobre a possível influência da ordem das questões de fecundidade nas respostas obtidas, assim como para outros temas específicos como, por exemplo, deficiência e rendimento. Ou seja, saber se perguntar sobre o número de nascidos vivos para cada uma das mulheres presentes no domicílio e depois fazer a pergunta sobre sobrevivência desses filhos, etc. (modelo de 2010), em vez de fazer todas as perguntas para uma mulher e depois repetir todas as perguntas para outra mulher do mesmo domicílio (modo antigo), causa vieses ou erros nas respostas. Ou se os controles possíveis de serem implementados no PDA, como não aceitar sobreviventes maior do que nascidos vivos, ou não perguntar por filhos 
nascidos no último ano para mulheres com zero filhos nascidos vivos, de fato controlam a qualidade da informação. Estas são questões que precisam ser investigadas com testes de campo ou análise de paradados, se disponíveis, antes da realização do próximo censo.

Outro tema importante para melhoria na qualidade é obter as informações sobre fecundidade quando o informante ainda não tenha se "cansado" da entrevista. o bloco de fecundidade no censo brasileiro sempre esteve no final do questionário. No caso de 2010, cujo questionário é bastante extenso, este fato pode ser prejudicial à qualidade da informação. Posicionar este bloco mais no início da entrevista e, ainda, mais próximo à própria lista de moradores do domicílio tem potencialidade para melhorar a qualidade da informação. Aqui também os testes antes do censo ir a campo são essenciais para analisar sua viabilidade e possíveis melhorias.

Para o cálculo da TFT, a boa qualidade da informação sobre FNUA é fundamental. Apesar de o questionário ter a pergunta sobre mês e ano de nascimento do último filho nascido vivo (e não somente se teve ou não nascido vivo no último ano), na base de dados é informada apenas a idade do último filho nascido vivo. Assim, para melhores testes sobre a qualidade desta informação, seria essencial disponibilizar na base de microdados a data completa de nascimento e a data da entrevista.

Finalmente, para se ter um panorama um pouco mais completo sobre a fecundidade da população brasileira e apontar tendências futuras, seria muito importante também coletar a informação sobre data de nascimento completa do primeiro filho nascido vivo. Com uma fecundidade já abaixo do nível de reposição e a moda do número de dois filhos, este procedimento possibilitaria ter a história completa de nascimento para mais da metade da população brasileira, fato que abriria horizontes para novas pesquisas e usos desta informação.

\section{Considerações finais}

Os dados de fecundidade no Censo Demográfico de 2010 apresentam boa qualidade da estrutura da fecundidade atual/corrente por idade das mulheres, mas encontram-se subestimados, como reconhecido pela literatura demográfica clássica. Ainda, o aumento da proporção de correção apresentado na série P/F em 2010 comparada a anos anteriores é coerente com uma pequena postergação da fecundidade em idades mais avançadas no período reprodutivo, não indicando, portanto, piora na qualidade dos dados. Dessa forma, é necessário efetuar o ajuste dos dados com a utilização de métodos indiretos. 0 nível do fator de ajuste é que, de fato, precisa ser investigado e, no caso do Brasil, o método P/F de Brass ainda se apresenta bastante adequado quando comparado com os dados do registro de nascimentos.

O valor correto da TFT em 2010, ou na média dos dois ou três anos anteriores, não pode ser motivo de disputa, visto que todos os dados e métodos disponíveis no momento apresentam algum tipo de problema. 0 que se pode fazer é buscar cada vez mais melhorar 
os dados do registro de nascimento e sua publicação oportuna para corrigir, no que for necessário, os dados coletados nas pesquisas domiciliares como o censo. Somente um registro administrativo com cobertura completa e de boa qualidade pode assegurar a estimativa do valor correto da fecundidade.

Também pode-se buscar melhorias na coleta dos dados censitários. Há necessidade de se fazerem testes mais eficientes para determinar a melhor forma de coletar os dados sobre fecundidade nos censos e pesquisas domiciliares em geral, pois é essencial conhecer estimativas mais acuradas, tanto da TFT quanto das taxas específicas por idade, para se projetar melhor estas estimativas que são fundamentais para as projeções populacionais, uma vez que a fecundidade, entre as três componentes demográficas, é a que ainda mais afeta o volume populacional. Uma diferença de um quarto de filho em uma taxa de fecundidade pode significar milhões de pessoas a mais no longo prazo em uma população de grande porte. Igualmente, a informação de boa qualidade sobre idade da população (e aqui das mulheres) é fundamental em uma pesquisa domiciliar. Logo, todo empenho em um censo para se obterem melhores informações sobre idade e fecundidade sempre será bem-vindo.

\section{Referências}

BERCOVICH, A. Sensibilidade das estimativas de fecundidade e mortalidade a alguns erros nas informações básicas. In: ENCONTRO NACIONAL DE ESTUDOS POPULACIONAIS, V. Anais... Águas de São Pedro: Abep, 1986. Disponível em: 〈http://www.abep.nepo.unicamp.br/docs/anais/ pdf/1986/T86V03A07.pdf >. Acesso em: 30 nov. 2015.

BERQUÓ, E.; CAVENAGHI, S. Notas sobre os diferenciais educacionais e econômicos da fecundidade no Brasil. Revista Brasileira de Estudos de População, São Paulo, v. 31, n. 2, p. 471-482, 2014. Disponível em: 〈http://dx.doi.org/10.1590/S0102-30982014000200012〉. Acesso em: 14 abr. 2016.

BRASS, W. Métodos para estimar la fecundidad y la mortalidad en poblaciones con datos limitados. Santiago, Chile: Centro Latinoamericano de Demografia, 1974.

CARVALHO, J. A. M. Aplicabilidade da técnica de fecundidade de Brass quando a fecundidade está declinando ou quando a população não é fechada. In: ENCONTRO NACIONAL DE ESTUDOS POPULACIONAIS, III. Anais... Vitória: Abep, 1982. Disponível em: 〈http://www.abep.nepo. unicamp.br/docs/anais/pdf/1982/T82V1A088.pdf>. Acesso em: 30 nov. 2015.

CAVENAGHI, S. A Spatial-temporal analysis of fertility transition and the health care delivery system in Brazil. Tese (Doutorado) - Universidade do Texas-Austin, Departamento de Sociologia, Texas-Austin, 1999. Disponível em: 〈http://www.ence.ibge.gov.br/ images/ence/ doc/PaginasProfessores/SuzanaCavenaghi/PhD_DissertationSuzana_Complete.pdf \. Acesso em: 30 nov. 2015.

CAVENAGHI, S.; ALVES, J. E. D. Domicilios y familias en la experiencia censal de Brasil: cambios y propuesta para identificar arreglos familiares. Notas de Población, v. 92, p. 15-46, 2011.

CHO, L. J. The own children approach to fertility estimation: an elaboration. In: INTERNATIONAL POPULATION CONFERENCE. Proceedings... Liege: IUSSP, 1973. v. 2.

CRUZ-CASTANHEIRA, H.; KOHLER, H. P. It is lower than you think it is: recent total fertility rates in Brazil and possibly other Latin American countries. In: POPULATION ASSOCIATION OF AMERICA. 
Proceedings... Washington, D.C, 2016. Disponivel em: 〈https://paa.confex.com/paa/2016/ mediafile/ExtendedAbstract/Paper4978/PAAfinal_Castanheira_Kohler_PFBrass.pdf \. Acesso em: 05 abr. 2016.

FERNANDEZ, R. E.; CARVALHO, J. A M. de. A evolução da fecundidade no Brasil, período 19571979. Aplicação da técnica dos filhos próprios para se estimar a fecundidade ano a ano. Revista Brasileira de Estudos de População, v. 3, n. 2, p. 67-86, 1986.

IBGE - Instituto Brasileiro de Geografia e Estatística. Censo Demográfico 2010: Manual do Recenseador. CD 1.09. Rio de Janeiro: IBGE, 2010.

Metodologia do Censo Demográfico 2010. Rio de Janeiro, 2013a. (Série Relatórios Metodológicos, v. 41). Disponível em: <ftp://ftp.ibge.gov.br/Censos/Censo_Demografico_2010/ metodologia/metodologia_censo_dem_2010.pdf>. Acesso em: 15 mar. 2014.

Projeções da população Brasil e Unidades da Federação. Rio de Janeiro, 2013b (Série Relatórios Metodológicos, v. 40).

MOULTRIE T. A.; DORRINGTON, R.; HILL, A.; HILL, K.; TIMAEUS, I.; ZABA, B. Tools for demographic estimation. Paris: International Union for the Scientific Study of Population, 2013. Disponivel em: 〈http://demographicestimation.iussp.org/〉. Acesso em: 13 jan. 2014.

MIRANDA-RIBEIRO, A. de; RIOS-NETO, E. L. G.; CARVALHO, J. A. de. Efeitos tempo, parturição e quantum no Brasil: indicadores de período e evidências empíricas. Revista Brasileira de Estudos de População, São Paulo, v. 30, n. 1, p. 145-170, 2013. Disponível em: 〈http://dx.doi. org/10.1590/S0102-30982013000100008>. Acesso em: 30 nov. 2015.

ONU - Organização das Nações Unidas. Métodos de estimação de medidas demográficas básicas a partir de informações incompletas: manual IV. Rio de Janeiro: IBGE, 1977.

SCHMERTMANN, C. P.; CAVENAGHI, S. M.; ASSUNÇÃO, R. M.; POTTER, J. E. Bayes plus Brass: estimating total fertility for many small areas from sparse census data. Population Studies, v. 67, n. 3, p. 225-273, 2013.

SCHMERTMANN C. P. Calibrated spline estimation of detailed fertility schedules from abridged data. Rostock: Max Planck Institute for Demographic Research, 2012 (MPIDR working paper, WP-2012-022). Disponivel em: 〈http://tinyurl.com/calibrated-spline〉. Acesso em: 13 jan. 2014.

UNITED NATIONS. The United Nations principles and recommendations for population and housing censuses: results of the survey on proposed changes for the 2020 census round. New York: Department of Economic and Social Affairs, Statistics Division, 2013. Disponivel em: 〈http:// unstats.un.org/unsd/demographic/meetings/egm/NewYork/2013/Country_suggestions.pdf . Acesso em: 29 out. 2013.

Manual X: indirect techniques for the demographic estimation, population studies, 81. New York: National Research Council, United States National Academy of Sciences, 1983.

WONG, L. R. Fecundidade no Brasil (urbano e rural) - aplicação do método dos filhos próprios ao Censo de 1970. Informe Demográfico, São Paulo, n. 9, 1983.

\section{Sobre os autores}

Suzana M. Cavenaghi é doutora em demografia pela Universidade do Texas-Austin e professora e pesquisadora da Escola Nacional de Ciências Estatísticas (Ence) do Instituto Brasileiro de Geografia e Estatística (IBGE), atuando no Programa de Pós-Graduação e População, Território e Estatísticas Públicas. 
José Eustáquio Diniz Alves é doutor em Demografia pelo Centro de Desenvolvimento e Planejamento Regional - Cedeplar da Universidade Federal de Minas Gerais - UFMG, com pós-doutorado no Núcleo de Estudos de População - Nepo, da Universidade Estadual de Campinas - Unicamp. Professor titular do Programa de Pós-graduação em População, Território e Estatísticas Públicas da Escola Nacional de Ciências Estatísticas - Ence, do Instituto Brasileiro de Geografia e Estatística - IBGE.

\title{
Endereço para correspondência
}

\author{
Suzana M. Cavenaghi \\ Escola Nacional de Ciências Estatísticas \\ Rua André Cavalcanti, 106, sala 502, Bairro de Fátima \\ 20231-050 - Rio de Janeiro-RJ \\ José Eustáquio Diniz Alves \\ Escola Nacional de Ciências Estatísticas \\ Rua André Cavalcanti, 106, sala 502, Bairro de Fátima \\ 20231-050 - Rio de Janeiro-RJ
}

\section{Abstract \\ Quality of information on fertility in the 2010 Population Census in Brazil}

The purpose of this research note is to exam the quality of information on fertility in the Brazilian demographic census of 2010 and to make inferences on the accuracy of the estimates of the total fertility rate (TFR) and age-specific rates estimated with that data source. The databases used are microdata from the 2010 population census and the Births Information System (Sinasc) of 2009 and 2010. Basic demographic indicators and the Brass' indirect method of P/F ratio to estimate TFR are used for the two data sources analyzed. The results show that census data are quite consistent, nonetheless they have problems of undercount for current fertility age-specific rates, as is well known in demography. This undercount must be taken into account when estimating the level of fertility for the country. The changes in fertility schedule affect fertility since TFR is a period rate, but the P/F Brass method still presents good results. Finally, some recommendations for possible improvements in data collection, mainly in censuses and sample survey of households are offered. These are essential data sources for estimating fertility and for monitoring its trends and for updating projections of this indicator in the medium and long run.

Keywords: Quality of information. Fertility. Indirect methods. Brazil.

\section{Resumen}

Calidad de las informaciones sobre fecundidad en el Censo Demográfico de 2010 de Brasil

El objetivo de esta nota de investigación es examinar la calidad de la información respecto la fecundidad en el censo demográfico brasileño de 2010, para inferir sobre la precisión de las estimativas de la tasa de fecundidad global (TFG) y de las tasas específicas por edad estimadas con esa fuente de datos. Las bases de datos utilizadas son los microdatos del censo demográfico de 2010 y el Sistema de Informaciones sobre Nacimientos (Sinasc) de 2009 y 2010. 
Se utilizan indicadores demográficos básicos y el método indirecto de la razón P/F de Brass para la estimación de la TFG, para las dos fuentes de datos analizadas. Los resultados muestran que los datos censales son bastante consistentes, más que presentan algunos problemas de subenumeración de la fecundidad corriente por edad, como es largamente conocido en la demografía. Esta subenumeración debe ser llevada en cuenta en la estimación del nivel de fecundidad para el país. Los cambios en la estructura de la fecundidad afectan la TFG por ser una tasa de periodo, pero el método P/F de Brass todavía presenta buenos resultados. Para finalizar, se presenta algunas recomendaciones de posibles mejoramientos en nuevos relevamientos de datos, principalmente en los censos y encuestas por muestreo de viviendas, que son fuentes esenciales para la estimación de la fecundidad y el seguimiento de sus tendencias para la necesaria actualización de las proyecciones de ese indicador a mediano y largo plazos.

Palabras clave: Calidad de la información. La fecundidad. Los métodos indirectos. Brasil.

Recebido para publicação em 30/03/2016 Recomendado para publicação em 30/03/2016

Aceito para publicação em 09/04/2016 
